\section{Associação entre atividade física e tempo de tela com o nível socioeconômico em adolescentes}

\section{Association between physical activity and screen time with socioeconomic level in adolescents}

Tatiane Motta da Costa e Silva'

Helter Luiz da Rosa Oliveira'

Mauren Lúcia de Araújo Bergmann

Gabriel Gustavo Bergmann

\section{RESUMO}

Apesar da reconhecida importância da atividade física (AF) para saúde, evidências sugerem que o tempo em atividades sedentárias tem aumentado entre adolescentes, podendo estar associado a diversos fatores, entre eles os socioeconômicos. O objetivo do estudo foi identificar as associações entre AF, comportamento sedentário e o nível socioeconômico (NSE) em adolescentes. $\mathrm{O}$ estudo contou com uma amostra de 1.455 adolescentes (741 moças) de 10 a 17 anos selecionados aleatoriamente em escolas públicas de Uruguaiana/RS. A AF foi estimada pelo $P A Q-C$ e $P A Q-A$ e os adolescentes foram classificados como suficientemente ou insuficientemente ativos. $\mathrm{O}$ comportamento sedentário foi considerado a partir do autorrelato (questionário) sobre o tempo de tela (tempo médio diário assistindo televisão, usando computador, videogame e outros equipamentos eletrônicos). Os adolescentes foram divididos em dois grupos: tempo de tela de até três horas/dia e superior a três horas/dia. O NSE foi considerado conforme o Critério de Classificação Econômica Brasil. Para a análise dos dados foi utilizada a estatística descritiva, o teste Qui-quadrado para tendência e a regressão logística binária. A AF não apresentou associação com o nível socioeconômico $(\mathrm{p}=0,562)$, inclusive quando a análise foi estratificada por sexo (rapazes $\mathrm{p}=0,360$; moças $\mathrm{p}=0,309$ ). O tempo de tela se associou ao nível socioeconômico apenas nos rapazes $(\mathrm{p}=0,043)$. Adolescentes do sexo masculino de nível socioeconômico mais alto têm mais chance (OR:1,83; IC95\%:1,02-3,28) de permanecer mais de três horas/dia em atividades sedentárias. O nível socioeconômico está diretamente associado ao tempo de tela em adolescentes do sexo masculino.

\section{PALAVRAS-CHAVE}

Atividade motora; Sedentarismo; Classe social.

\begin{abstract}
Despite the recognized importance of physical activity (PA) for health, evidence suggests that the time in sedentary activities has increased among adolescents and may be associated with several factors, including socioeconomic. The aim of the study was to identify associations between AF, sedentary behavior and socioeconomic status (SES) in adolescents. The study had a sample of 1,455 adolescents (741 girls) from 10 to 17 years randomly selected from public schools in Uruguaiana/RS. The AF was estimated by the $P A Q-C$ and $P A Q-A$ and adolescents were classified as sufficiently or insufficiently active. Sedentary behavior was considered from the self-report (questionnaire) on screen time (average daily time watching television, using computer, video games and other electronic equipment). The adolescents were divided into two groups: screen time up to three hours / day and more than three hours / day. The NSE was considered as the Criterion of Economic Classification Brazil. For data analysis was used descriptive statistics, Chi-square test for trend and binary logistic regression. The AF was not associated with socioeconomic status $(p=0.562)$, even when the analysis was stratified by gender $(p=0.360$ boys; girls $p=0.309)$. The screen time was associated with socioeconomic level only in boys $(p=0.043)$. Male adolescents of higher socioeconomic status are more likely (OR: 1.83; $95 \%$ CI: 1.02 to 3.28) to stay more than three hours / day in sedentary activities. Socioeconomic status is directly associated with the screen time in male adolescents.
\end{abstract}

\section{KEYWORDS}

Motor Activity; sedentary lifestyle; Social Class.
Rev Bras Ativ Fís Saúde p. 503-513 DOI

http://dx.doi.org/10.12820/rbafs.v.20n5p503

1 Universidade Federal do Pampa - Grupo de Pesquisa em Atividade Física e Saúde na Infância e Adolescência, Curso de Licenciatura em Educação Física, Uruguaiana, Rio Grande do Sul, Brasil. 


\section{INTRODUÇÃOO}

A prática regular de Atividade Física $(\mathrm{AF})$ resulta em diversos benefícios à saúde e qualidade de vida da população, além de ser um importante fator de prevenção contra inúmeras doenças ${ }^{1}$. Em crianças e adolescentes estes benefícios podem ser identificados em curto e longo prazo. Estudos transversais têm sugerido que crianças e adolescentes mais ativos têm menos chance de serem obesos $^{2}$ e de apresentarem pressão arterial elevada ${ }^{3}$ e hipercolesterolemia ${ }^{4}$. Em relação aos benefícios em longo prazo, indivíduos ativos durante a infância e adolescência tendem a ser mais ativos e ter menos chance de desenvolverem osteoporose e determinados tipos de câncer na idade adulta 5 .

Apesar da reconhecida importância da prática de AF durante a infância e adolescência, a quantidade de jovens que atinge as recomendações relativas à quantidade e intensidade de prática é baixa ${ }^{6}$ e parece estar diminuindo entre adolescentes brasileiros nos últimos anos ${ }^{7,8}$. Por outro lado, o número de jovens que preenche parte significativa de seu tempo diário com atividades sedentárias é elevado ${ }^{9,10}$ e com evidências de estar aumentando nos últimos $\operatorname{anos}^{10}$. Tais evidências são preocupantes já que o aumento no comportamento sedentário parece estar positivamente associado à obesidade ${ }^{11}$, ao consumo de álcool, hábitos alimentares não saudáveis e a prejuízos em determinados indicadores de saúde psicossocial ${ }^{12}$. Devido aos possíveis prejuízos à saúde associados ao baixo nível de atividade física ${ }^{2-5}$ e ao comportamento sedentário ${ }^{11,12}$ já nos anos da infância e adolescência, essa situação deve ser amplamente investigada para que ações sejam elaboradas com o intuito de alterar este quadro.

Diversos fatores precisam ser considerados para a melhor compreensão dos motivos que levam crianças e adolescentes a adotarem comportamentos ativos e sedentários em seu dia a dia. O nível socioeconômico (NSE) familiar é um fator importante a ser avaliado. Nesta perspectiva, alguns estudos vêm sendo conduzidos associando indicadores socioeconômicos com a AF e o comportamento sedentário da população jovem. Os resultados encontrados são conflitantes ${ }^{13-18}$, não permitindo identificar claramente como estas variáveis se associam, necessitando que mais investigações com este propósito sejam conduzidas. Esta heterogeneidade nos resultados pode estar relacionada a diferenças metodológicas entre os estudos, incluindo os procedimentos de seleção das amostras e os instrumentos de medida das variáveis. Além disto, poucos foram os estudos que realizaram as análises estratificadas por sexo ${ }^{14,18}$. Considerando que adolescentes do sexo masculino são mais ativos ${ }^{13,14,17,19} \mathrm{e}$ tendem a permanecer menos tempo em atividades sedentárias ${ }^{15-19}$ que adolescentes do sexo feminino, análises envolvendo fatores associados, como indicadores socioeconômicos, a estes dois desfechos estratificadas por sexo devem ser realizadas. Resultados destas análises podem trazer informações para a elaboração de estratégias de aumento dos níveis de AF e redução do tempo em atividades sedentárias específicas para o sexo masculino e feminino. $\mathrm{O}$ presente estudo tem como objetivo identificar as possíveis associações entre AF, comportamento sedentário e o NSE em adolescentes dos sexos masculino e feminino. 


\section{MÉTODOS}

\section{População e amostra}

Este estudo transversal descritivo foi elaborado com informações de adolescentes de do município de Uruguaiana/RS. Distante cerca de 630 quilômetros da capital Porto Alegre, o município de Uruguaiana se localiza no extremo oeste do estado do Rio Grande do Sul, na fronteira com a Argentina. De acordo com os dados do censo demográfico de 2010 do Instituto Brasileiro de Geografia e Estatística (IBGE), Uruguaiana possui uma população de 125.435 habitantes e uma área de 5.715,763 quilômetros quadrados, tendo a agricultura e a pecuária como suas as principais atividades econômicas.

A amostra contou com informações de 1.455 (50,9\% moças) adolescentes do banco de dados do projeto "Atividade física e fatores associados em escolares de 10 a 17 anos de Uruguaiana, RS". O projeto foi aprovado pelo comitê de ética em pesquisa da Universidade Federal do Pampa (protocolo 042/2010). O cálculo do tamanho mínimo da amostra para a realização do projeto considerou o número de alunos (15.210), na faixa etária do estudo, matriculados regularmente no ano de 2011 de acordo com informações das secretarias estadual e municipal de educação; prevalência de $50 \%$, considerando um estudo com múltiplos desfechos; intervalo de confiança de $95 \%$; erro aceitável de 3 pontos percentuais; e acréscimo de 20\% para compensar possíveis perdas e recusas. Com a adoção destes critérios foi estimada a necessidade de selecionar 1.196 escolares.

O processo de seleção dos adolescentes foi probabilístico multifásico. Com o intuito de manter a representatividade e proporcionalidade de alunos por regiões, inicialmente o município foi dividido em cinco áreas geográficas, quatro na zona urbana e zona rural como um todo. Após, foi identificado o número de escolas e de alunos, na faixa etária de 10 a 17 anos, matriculados em cada área. Para alcançar o número estimado de adolescentes da zona urbana houve a necessidade de sortear duas escolas em três das quatro áreas geográficas e três escolas na quarta área, totalizando nove escolas urbanas. Para a seleção dos alunos da zona rural foi necessário sortear, entre todas as escolas desta zona, apenas uma escola. Após a definição das escolas, representantes de cada uma delas foram contatados para a apresentação dos objetivos e procedimentos do estudo e permitiram a sua realização. Todos os escolares de 10 a 17 anos das 10 escolas foram convidados a participar. Os critérios de inclusão utilizados foram: a) estar matriculado e frequentando as aulas na rede pública de ensino (estadual ou municipal) do município; e, b) Apresentar o termo de consentimento livre e esclarecido assinado por um responsável e manifestar vontade em participar. Foram excluídos os adolescentes fora da faixa etária de interesse e aqueles que apresentassem algum tipo de deficiência física ou mental que os impedissem de praticar atividades físicas e/ou de compreenderem a realização das medidas. Os dados foram coletados no período de abril a novembro de 2011.

Considerando que o cálculo de tamanho de amostra do projeto foi conduzido para estimar uma prevalência de $50 \%$ com uma margem de erro de 3 pontos percentuais, tendo em vista seus diferentes objetivos, o poder da amostra para as análises deste estudo, considerando a estratificação por sexo, foi mensurado a posteriori. Assim, a amostra analisada permitiu estimar pre- 
valências de $10,5 \%$ a $80,5 \%$, com erros de 1,5 a 3,5 pontos percentuais, respectivamente, $(\mathrm{P}<0,05)$. Para as medidas de associação, a amostra tem poder de $80 \%(\beta=20 \%)$ e nível de confiança de $95 \%(\alpha=5 \%)$ para detectar razões de chances (OR) iguais ou superiores a 1,31 como significativas.

\section{Variáveis e procedimentos de coleta de dados}

Para a elaboração deste estudo foram utilizadas informações sobre o NSE, o comportamento sedentário e o nível de AF. Estas informações foram coletadas em sala durante o período de aula. Todos os escolares recebiam uma cópia do instrumento e orientações sobre o seu preenchimento. Membros da equipe avaliadora ficavam à disposição para esclarecimentos de eventuais dúvidas. Além disto, com o intuito de evitar a perda de informações, identificavam se todas as questões estavam preenchidas.

O NSE foi estimado com o instrumento de Classificação Econômica Brasil $^{20}$ e sua classificação em cinco níveis de "A" a "E". O comportamento sedentário foi considerado a partir do autorrelato dos adolescentes sobre o tempo médio diário de tela (utilizando televisão, jogos eletrônicos, computador e outros equipamentos eletrônicos). A partir destas informações os adolescentes foram classificados em dois grupos: aqueles que relataram passar em média mais de três horas ( $>3$ horas) por dia nas atividades citadas e que relataram passar em média até três horas ( $\leq 3$ horas). A AF foi estimada por meio do questionário para atividades físicas para crianças e a adolescentes (Physical Activity Questionnaire for Older Children PAQ-C $C^{21}$ and Adolescents $\left.P A Q-A^{22}\right)$. Os resultados são expressos em um escore contínuo que, dividido pelo número de questões, permite a criação de cinco categorias de AF, classificando os indivíduos em muito sedentário, sedentário, moderadamente ativo, ativo ou muito ativo, conforme a proposta original ${ }^{21,22}$. Entretanto, em nossos estudos optamos por agrupar e substituir as expressões muito sedentário e sedentário por insuficientemente ativos, e agrupar as expressões moderadamente ativo, ativo e muito ativo em suficientemente ativos. Tal escolha ocorreu por, assim como outros autores ${ }^{23}$, entendermos que um indivíduo mesmo com comportamentos sedentários, ou com um estilo de vida sedentário, pode ser considerado ativo, visto que o termo sedentarismo remete ao tempo diário despendido na posição sentada, e não a ausência de prática de atividade física.

Além das descritas anteriormente, também foram utilizadas as variáveis sexo (masculino ou feminino), idade (anos completos) e zona de moradia (rural ou urbana). Elas foram utilizadas nas análises estratificadas (sexo) e na análise multivariável como variáveis de ajuste.

\section{Análise dos dados}

Para a análise estatística inicialmente foi realizada a descrição dos dados com a utilização das frequências absolutas e relativas (proporções) em cada uma das variáveis analisadas seguidas pelo cálculo do intervalo de confiança de 95\% (IC95\%). Para a identificação das frequências de adolescentes insuficientemente ativos e com tempo de tela $>3$ horas nas categorias do NSE foi utilizado os testes Qui-quadrado para tendência linear. Por fim, foi utilizada a regressão logística binária tendo a $\mathrm{AF}$ e o comportamento sedentário dicotomizados como desfecho, NSE como variável independente e o sexo, a idade 
e a zona de moradia como variáveis de ajuste. Nesta análise a inserção das variáveis independente e de ajuste foi realizada utilizando o método enter em um único bloco. As análises do teste Qui-quadrado e da regressão logística binária foram conduzidas com toda a amostra e estratificadas por sexo. O nível de significância adotado foi de $5 \%$ e todas as análises foram realizadas no SPSS for Windows versão 20.0 .

\section{RESULTADOS}

Do total de adolescentes selecionados (1.554) para participar do estudo, 41 não apresentaram o termo de consentimento livre e esclarecido assinado por um responsável ou não estiveram presentes no dia da coleta dos dados e 50 não quiseram participar do estudo. Dentre os avaliados (1.463), oito não fizeram parte das análises por terem menos do que 10 anos ou mais de 17 anos de idade. Do total de adolescentes que tiveram suas informações coletadas (1.455), 1.3434 e 1.278 preencheram os questionários de AF e NSE, respectivamente, de forma que estas variáveis pudessem ser classificadas. A tabela 1 apresenta a descrição do nível de AF, do tempo de tela e das características sociodemográficas da amostra analisada.

TABELA 1 - Descrição do nível de atividade física, tempo de tela e de características sociodemográficas de adolescentes de 10 a 17 anos. Uruguaiana/RS, 2011.

\begin{tabular}{|c|c|c|c|c|c|c|}
\hline \multirow[b]{2}{*}{ Variável } & \multicolumn{2}{|c|}{ Toda amostra } & \multicolumn{2}{|r|}{ Masculino } & \multicolumn{2}{|r|}{ Feminino } \\
\hline & $n$ & $\%$ (IC95\%) & $n$ & $\%$ (IC95\%) & $\mathrm{n}$ & \% (IC95\%) \\
\hline \multicolumn{7}{|l|}{ Atividade Física } \\
\hline Suficientemente ativo & 430 & $32,0(27,6-36,4)$ & 311 & $46,9(43,1-50,7)$ & 119 & $17,5(14,6-20,4)$ \\
\hline Insuficientemente ativo & 913 & $68,0(65,0-71,0)$ & 352 & $53,1(49,3-56,9)$ & 561 & $82,5(79,6-85,4)$ \\
\hline \multicolumn{7}{|l|}{ Tempo de tela } \\
\hline$\leqslant 3$ horas & 521 & $35,8(33,3-38,3)$ & 273 & $38,2(34,6-41,8)$ & 248 & $33,5(30,1-36,9)$ \\
\hline$>3$ horas & 934 & $64,2(61,7-66,7)$ & 441 & $61,8(58,2-65,4)$ & 493 & $66,5(63,1-69,9)$ \\
\hline \multicolumn{7}{|l|}{ Nível Socioeconômico } \\
\hline B & 263 & $20,6(14,3-18,3)$ & 132 & $21,4(18,2-24,6)$ & 131 & $19,8(16,8-22,8)$ \\
\hline C & 817 & $63,9(61,3-66,5)$ & 400 & $64,9(61,1-68,7)$ & 417 & $63,0(59,3-66,7)$ \\
\hline D & 190 & $14,9(13,0-16,8)$ & 81 & $13,1(10,4-15,8)$ & 109 & $16,5(13,7-19,3)$ \\
\hline E & 8 & $0,6(0,2-1,0)$ & 3 & $0,5(0,3-0,7)$ & 5 & $0,8(5,9-10,1)$ \\
\hline \multicolumn{7}{|l|}{ Zona de residência } \\
\hline Urbana & 1296 & $90,8(89,3-92,3)$ & 649 & $90,9(88,8-93,0)$ & 675 & $91,1(89,1-93,1)$ \\
\hline Rural & 131 & $9,2(7,7-10,7)$ & 65 & $9,1(7,0-11,2)$ & 66 & $8,9(6,9-10,9)$ \\
\hline \multicolumn{7}{|l|}{ Idade } \\
\hline 10 anos & 79 & $5,4(4,2-6,6)$ & 41 & $5,7(4,0-7,4)$ & 38 & $5,1(3,5-6,7)$ \\
\hline 11 anos & 209 & $14,4(12,6-16,2)$ & 97 & $13,6(11,1-16,1)$ & 112 & $15,1(12,5-17,7)$ \\
\hline 12 anos & 205 & $14,1(12,3-15,9)$ & 102 & $14,3(11,7-16,9)$ & 103 & $13,9(11,4-16,4)$ \\
\hline 13 anos & 232 & $15,9(14,0-17,8)$ & 122 & $17,1(14,3-19,9)$ & 110 & $14,8(12,2-17,4)$ \\
\hline 14 anos & 211 & $14,5(12,7-16,3)$ & 104 & $14,6(12,0-17,2)$ & 107 & $14,4(11,9-16,9)$ \\
\hline 15 anos & 221 & $15,2(13,3-17,1)$ & 101 & $14,1(11,6-16,6)$ & 120 & $16,2(13,6-18,8)$ \\
\hline 16 anos & 174 & $12,0(10,3-13,7)$ & 85 & $11,9(9,5-14,3)$ & 89 & $12,0(9,7-14,3)$ \\
\hline 17 anos & 124 & $8,5(7,1-9,9)$ & 62 & $8,7(6,6-10,8)$ & 62 & $8,4(6,4-10,4)$ \\
\hline
\end{tabular}

n: tamanho da amostra; \%: proporção da amostra; IC95\%: intervalo de confiança de $95 \%$. 
Nenhum adolescente foi classificado dentro do NSE "A". Além disto, devido ao reduzido número de adolescentes classificados no NSE "E", esta categoria foi agrupada à categoria "D”. Assim, para as análises de associação o NSE foi considerado com três categorias (B, C e D/E). A frequência de adolescentes insuficientemente ativos e com tempo de tela $>$ três horas diárias não diferiu entre as categorias do NSE ( $>00,05)$. Quando as análises são estratificadas por sexo, o tempo de tela entre os rapazes aumentou significativamente $(\mathrm{p}<0,05)$ à medida que o NSE também aumentou (figura 1$)$.

$\mathrm{B} \square \mathrm{C} \square \mathrm{D} / \mathrm{E}$

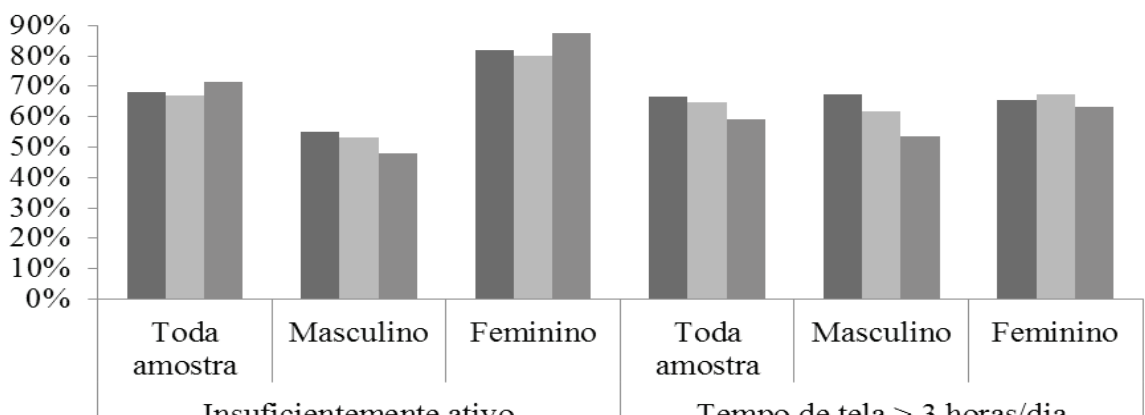

FIGURA 1 - Frequência de adolescentes insuficientemente ativos e com tempo de tela superior a três horas diárias segundo o nível socioeconômico (A, B e D/E). Uruguaiana/RS, 2011.

As análises da regressão logística binária (tabelas 2 e 3), mesmo após ajuste para variáveis demográficas, confirmaram os resultados encontrados nos testes de Qui-quadrado. Somente o tempo de tela entre os rapazes apresentou associação estatisticamente significativa $(\mathrm{p}=0,044)$ com o NSE. Adolescentes do sexo masculino de NSE mais alto (B) têm $83 \%$ mais chance de terem um tempo de tela médio maior de três horas por dia quando comparados àqueles de NSE mais baixo (D/E).

TABELA 2 - Associação bruta entre o nível de atividade física e o tempo de tela com o nível socioeconômico de adolescentes de 10 a 17 anos. Uruguaiana/RS, 2011.

\begin{tabular}{|c|c|c|c|c|c|c|}
\hline & \multicolumn{3}{|c|}{ Insuficientemente ativo } & \multicolumn{3}{|c|}{ Tempo de tela $>3$ horas/dia } \\
\hline & Toda amostra & Masculino & Feminino & Toda amostra & Masculino & Feminino \\
\hline & OR (IC95\%) & OR (IC95\%) & OR (IC95\%) & OR (IC95\%) & OR (IC95\%) & OR (IC95\%) \\
\hline \multicolumn{7}{|c|}{ Nível Socioeconômico } \\
\hline B & $0,86(0,56-1,31)$ & $1,34(0,75-2,39)$ & $0,58(0,31-1,08)$ & $1,38(0,94-2,02)$ & $1,79(1,02-3,15)$ & $1,12(0,66-1,88)$ \\
\hline C & $0,81(0,56-1,16)$ & $1,24(0,74-2,05)$ & $0,64(0,31-1,35)$ & $1,26(0,92-1,74)$ & $1,40(0,87-2,25)$ & $1,21(0,78-1,86)$ \\
\hline $\mathrm{D} / \mathrm{E}$ & 1 & 1 & 1 & 1 & 1 & 1 \\
\hline
\end{tabular}

OR: razão de odds; IC95\%: intervalo de confiança de 95\%.

TABELA 3 - Associação ajustada entre o nível de atividade física e o tempo de tela com o nível socioeconômico de adolescentes de 10 a 17 anos. Uruguaiana/RS, 2011.

\begin{tabular}{lcccccc}
\hline & \multicolumn{3}{c}{ Insuficientemente ativo } & \multicolumn{3}{c}{ Tempo de tela > 3 horas/dia } \\
\hline & Toda amostrat & Masculino* & Feminino* & Toda amostrat & Masculino* & Feminino* \\
\hline & OR (IC95\%) & OR (IC95\%) & OR (IC95\%) & OR (IC95\%) & OR (IC95\%) & OR (IC95\%) \\
\hline Nível Socioeconômico & \multicolumn{7}{c}{ OC95) } & & & & \\
\hline B & $1,01(0,63-1,63)$ & $1,39(0,75-2,55)$ & $0,66(0,30-1,49)$ & $1,43(0,96-2,13)$ & $1,83(1,02-3,28)$ & $1,13(0,65-1,96)$ \\
\hline C & $0,86(0,57-1,28)$ & $1,22(0,72-2,07)$ & $0,53(0,27-1,05)$ & $1,29(0,93-1,79)$ & $1,40(0,86-2,29)$ & $1,19(0,76-1,86)$ \\
\hline D/E & 1 & 1 & 1 & 1 & 1 & 1 \\
\hline
\end{tabular}

†: Ajustada para sexo, idade e zona de moradia; *Ajustada para idade e zona de moradia; OR: razão de odds; IC95\%: intervalo de confiança 


\section{DISCUSSÃO}

O principal resultado do presente estudo foi identificar que o tempo de tela está diretamente associado ao NSE em adolescentes do sexo masculino. Além deste, os resultados referentes às frequências de adolescentes insuficientemente ativos e com tempo de tela superior a três horas diárias devem ser destacados devido a sua elevada prevalência.

Embora a proporção de adolescentes insuficientemente ativos tenha sido elevada, resultados de estudos brasileiros utilizando a mesma forma de medida e classificação da AF apresentaram prevalências ainda mais altas ${ }^{13,17}$. Outras investigações com amostras populacionais que utilizaram outros instrumentos de medida e como critério para a classificação alcançar no mínimo 300 minutos por semana de $\mathrm{AF}$ de intensidade moderada a vigora, também encontraram prevalências elevadas de adolescentes insuficientemente ativos, variando entre $49,8 \%$ e $80,3 \%, 9,14,19,26$.

As diferenças encontradas nas prevalências de adolescentes classificados como insuficientemente ativos entre os estudos podem estar associadas a diferentes fatores. A utilização de instrumentos e procedimentos de classificação distintos associados a características sazonais, ambientais e culturais, dificultam a comparação e a interpretação das diferenças encontradas entre os estudos. No entanto, é importante destacar que independente das características citadas, todos os estudos apresentaram elevadas proporções de adolescentes insuficientemente ativos. Este panorama se torna ainda mais preocupante ao se considerar as informações sobre AF divulgadas pela Pesquisa Nacional de Saúde do Adolescente dos anos de $2009^{7}$ e de $2012^{8}$. Em três anos houve uma redução de $13 \%$ na quantidade de adolescentes considerados suficientemente ativos nas capitais brasileiras e no Distrito Federal.

Quanto ao comportamento sedentário, no presente estudo 64,2\% dos jovens relataram um tempo de tela médio superior a três horas por dia. Confrontando estes resultados aos disponíveis na literatura, proporções mais baixas $^{15,19}$, próximas ${ }^{17,18}$ e mais altas ${ }^{9,16}$ de adolescentes expostos ao comportamento sedentário são encontradas. Similar ao discutido para $\mathrm{AF}$, a variação nos resultados referentes ao comportamento sedentário pode ser atribuída a diferenças culturais e ambientais das regiões onde os estudos foram conduzidos. Além disto, tais diferenças podem estar associadas à forma com que este comportamento foi medido e classificado. Da mesma forma que o presente, alguns estudos também consideraram o comportamento sedentário a partir do somatório de tempo dispendido assistindo televisão, jogando videogame e utilizando computador ${ }^{9,15,16}$. No entanto, outros consideraram apenas o tempo assistindo televisão $0^{17,18,19}$. Além disto, o critério para a classificação dos adolescentes expostos ao comportamento sedentário variou entre duas ${ }^{9,16}$, três ${ }^{17,19}$ e quatro horas por dia ${ }^{15}$. Esta diversidade de características entre os estudos compromete a comparação e interpretação de seus resultados. Contudo, independente das diferenças encontradas, nota-se uma alta prevalência de jovens expostos ao comportamento sedentário.

As elevadas prevalências de adolescentes insuficientemente ativos e expostos a comportamento sedentário encontrados no presente estudo e entre os citados alertam para que atitudes sejam tomadas na tentativa de reverter essa situação. A necessidade de que mais ações sejam elaboradas para rever- 
ter este quadro é urgente. Para isto, é importante que fatores associados a níveis insuficientes de AF e a comportamentos sedentários em adolescentes sejam considerados. No presente estudo, foram investigadas as possíveis associações do NSE com estes dois comportamentos.

Os achados referentes às análises entre a AF e o NSE indicaram não existir associação entre as variáveis, inclusive quando a análise é estratificada por sexo. Estes resultados também foram encontrados por outros estudos ${ }^{13,24,27}$. De forma semelhante, Farias Júnior et al. ${ }^{14}$ também não encontraram associação para sexo masculino. Para o sexo feminino, contudo, os autores encontraram, mesmo sem uma tendência linear clara, que as moças pertencentes à classe econômica $\mathrm{C}$ eram mais propensas a ser fisicamente mais ativas quando comparadas às da classe econômica $\mathrm{E}$. No entanto, existem evidências de uma série de investigações indicando que níveis insuficientes de AF estão as-

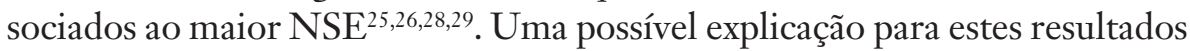
está no fato de que famílias de maior poder aquisitivo podem proporcionar a seus filhos o acesso a opções de lazer com caráter mais sedentário, como vídeo games, computadores e suas variações (tablets e telefone celulares inteligentes) com acesso ilimitado a internet. No entanto, é importante destacar que estudos que analisaram a $\mathrm{AF}$ em seus diferentes domínios indicaram que maiores níveis de $\mathrm{AF}$ no lazer estão associados aos adolescentes de maior $\mathrm{NSE}^{30}$, enquanto que maiores quantidades de deslocamento ativo estão associados aos adolescentes de menor $\mathrm{NSE}^{25}$. A falta de padronização nas formas de medida e classificação da AF e do NSE não permite uma clara definição sobre as associações entre estas variáveis, sendo uma possível razão para a inconsistência nos resultados encontrados entre os estudos.

Quanto ao comportamento sedentário e o NSE, na análise envolvendo toda a amostra, não foi identificada associação significativa. Esse resultado se assemelha ao de outros estudos ${ }^{16,17}$. Contudo, as investigações conduzidas por Dias et al. ${ }^{15}$ e Oliveira et al. ${ }^{27}$ sugerem que um maior tempo de tela é mais frequente entre os adolescentes de maior NSE. A presença de um NSE econômico familiar mais elevado pode facilitar o acesso a tecnologias e assim favorecer o aumento do comportamento sedentário, principalmente entre a população jovem. No entanto, devido a diversidade nos resultados disponibilizados pelos estudos citados acima, não há consenso sobre essa temática. Diferenças em características culturais e ambientais inerentes às regiões onde os estudos foram desenvolvidos, bem como diferenças metodológicas como a forma de caracterização do comportamento sedentário e os pontos de corte utilizados, podem influenciar para que não haja homogeneidade nos resultados. Torna-se necessária a elaboração de novos estudos para que seja possível uma melhor análise, comparação e compreensão dos resultados, possibilitando o desenvolvimento de estratégias efetivas para redução do tempo de adolescentes em atividades sedentárias.

Dentre os estudos disponíveis na literatura, são escassos aqueles que realizaram análises estratificadas por sexo entre o comportamento sedentário e o NSE, dificultando a comparação e interpretação dos resultados. No presente estudo a frequência de rapazes que relatou permanecer exposto ao tempo de tela superior a três horas foi significativamente superior entre aqueles de maior NSE. No estudo de Silva et al. ${ }^{18}$, por outro lado, foi encontrada associação entre o maior tempo assistindo televisão e menor NSE entre as 
meninas. A diferença encontrada nestes resultados pode estar associada aos procedimentos de medida do comportamento sedentário.

Considerando que o uso de vídeo games é mais frequente no sexo masculino ${ }^{16}$ e que os aparelhos mais modernos e que mais atraem os adolescentes tem um custo elevado, estas características podem auxiliar na compreensão da razão para no presente estudo um maior tempo de tela entre os rapazes de maior NSE ter sido encontrado. No estudo de Silva et al. ${ }^{18}$ o comportamento sedentário foi considerado apenas a partir do tempo assistindo televisão. Como este equipamento eletrônico se popularizou nos últimos anos entre famílias de menor poder aquisitivo e a frequência de moças que relatou assistir televisão por mais de duas horas por dia foi superior à encontrada entre os rapazes, estas características contribuem para uma possível explicação no resultado encontrado.

Mais estudos analisando as associações entre comportamento sedentário e NSE em adolescentes estratificados por sexo devem ser realizadas. Análises envolvendo as diferentes possibilidades de tempo de tela (assistir televisão, jogar vídeo game e utilizar computador) separadamente devem ser conduzidas, pois parece estar havendo uma redução no tempo assistindo televisão e aumento no tempo utilizando computadore ${ }^{10}$. Ainda, devido ao rápido aumento do uso de telefones inteligentes entre adolescentes, esta característica também deve ser considerada. Além disto, analisar apenas o tempo de tela para identificar o quanto os adolescentes estão expostos ao comportamento sedentário pode ser insuficiente. Outros domínios desse comportamento podem preencher parte importante do tempo diário destes indivíduos e, desta forma, também devem ser considerados em novos estudos envolvendo o comportamento sedentário.

Os resultados encontrados, embora com divergências em relação a outros utilizados nesta discussão, são fortalecidos por características metodológicas desta investigação. $\mathrm{O}$ tamanho da amostra e sua representatividade, que contou com a participação de adolescentes da zona rural e de diferentes áreas da zona urbana, oferece maior poder às análises e validade interna ao estudo. A realização de análises estratificadas por sexo nas associações entre AF, comportamento sedentário e o NSE é outra aspecto positivo, contribuindo para melhor compreensão destas associações em moças e rapazes.

Não obstante os aspectos positivos, algumas limitações e suas possíveis repercussões devem ser discutidas. Apesar do estudo ter forças para generalizações em sua população (validade interna), a generalização para a população de adolescentes em geral (validade externa) merece cautela. A associação entre o NSE e os desfechos analisados pode variar devido a características culturais inerentes às populações analisadas. Isto fortalece a necessidade de estudos em diferentes regiões. $\mathrm{O}$ fato de nenhum adolescente ter sido classificado como pertencente à classe econômica "A" pode ter influenciado nos resultados e poderia ter sido minimizada se adolescentes de escolas privadas tivessem sido incluídos no estudo. $\mathrm{O}$ uso de informações autorrelatadas pelos adolescentes para a estimativa da $\mathrm{AF}$, do comportamento sedentário e do NSE configura-se como outra limitação, pois está associado ao viés de memória podendo influenciar nos resultados destas variáveis. Além disto, não foi testada a reprodutibilidade das informações. Tanto o comportamento sedentário quanto a $\mathrm{AF}$ foram estimados sem considerar diferenças entre os 
dias de semana e o final de semana. Como estes comportamentos podem variar neste período, esta é outra limitação deste estudo. Ainda, se o comportamento sedentário, além de ter sido analisado pelo somatório do tempo médio diário assistindo televisão, jogando vídeo game, tivesse sido analisado com os três comportamentos separadamente, outros resultados poderiam ter sido encontrados. Ademais, como comentado anteriormente, apenas o tempo de tela pode não refletir o tempo total em atividades sedentárias, havendo a necessidade de que outros domínios deste comportamento sejam considerados.

Diante dos resultados encontrados, pode-se concluir que a população investigada apresenta uma alta prevalência de adolescentes insuficientemente ativos e expostos a comportamentos sedentários. O NSE não se associa à $\mathrm{AF}$, mesmo quando estratificado por sexo. No que se refere ao comportamento sedentário, o tempo de tela diário está diretamente associado ao NSE em adolescentes do sexo masculino, indicando que as ações para a redução do comportamento sedentário entre adolescentes devem considerar o NSE, particularmente entre os rapazes. Novos estudos envolvendo as associações entre NSE, a AF e o comportamento sedentário devem ser conduzidos. Estes estudos devem considerar a utilização de diferentes indicadores socioeconômicos e em relação à $\mathrm{AF}$ e ao comportamento sedentário considerar diferentes domínios.

\section{Contribuição dos autores}

TMCS e HLRO participaram da análise e interpretação dos dados, da redação do artigo e aprovaram a versão final ser publicada. MLAB e GGB participaram de todas as etapas do estudo e aprovaram sua versão final.

\section{REFERÊNCIAS}

1. Lee IM, Shiroma EJ, Lobelo F, Puska P, Blair SN, Katzmarzyk PT. Effect of physical inactivity on major non-communicable diseases worldwide: in analysis of burden of disease and life expectancy. Lancet. 2012;380:219-29.

2. Bozza R, Campos W, Bacil ED, Barbosa Filho VC, Hardt JM, da Silva PM. Fatores sociodemográficos e comportamentais associados à adiposidade corporal em adolescentes. Rev Paul Ped. 2014;32(3):241-6.

3. Guedes DP, Guedes JERP, Barbosa DS, Oliveira, JAD, Stanganelli LCR. Fatores de risco cardiovasculares em adolescentes: indicadores biológicos e comportamentais. Arq Bras Cardiol. 2006;86(6):439-50.

4. Romero A, Medeiros MJ, Borges CA, Romero SS, Slater B. Associação entre atividade física e marcadores bioquímicos de risco para doença cardiovascular em adolescentes de escolas públicas de Piracicaba. Rev Bras Ativ Fís Saúde. 2013;18(5):614-22.

5. Hallal PC, Victora CG, Azevedo MR, Wells JC. Adolescent physical activity and health: a systematic review. Sports Med. 2006;36:1019-30.

6. Hallal PC, Andersen LB, Bull FC, Regina G, Haskell W, Ekelund U. Physical Activity Series Working Group. Global physical activity levels: surveillance progress, pitfalls, and prospects. Lancet. 2012;380(9838):247-57

7. Instituto Brasileiro de Geografia e Estatística. Pesquisa Nacional de Saúde Escolar. Rio de Janeiro: IBGE; 2009.

8. Instituto Brasileiro de Geografia e Estatística - IBGE (Brasil). Pesquisa Nacional de Saúde do Escolar - PENSE 2012. Rio de Janeiro: IBGE; 2013

9. Coelho LG, Cândido APC, Machado-Coelho GL, Freitas SND. Associação entre estado nutricional, hábitos alimentares e nível de atividade física em escolares. J. Pediatr. 2012;88(5):406-12.

10. Bucksch J, Inchley J, Hamrik Z, Finne E, Kolip P, HBSC. Study Group Germany. Trends in television time, non-gaming PC use and moderate-to-vigorous physical activity among German adolescents 2002-2010. BMC Public Health. 2014;14(1):351. 
11. Dumith SC, Garcia LM, da Silva KS, Menezes AM, Hallal PC. Predictors and health consequences of screen-time change during adolescence-1993 Pelotas (Brazil) birth cohort study. J Adolesc Health. 2012;51(6 Suppl):S16-21.

12. Busch V, Manders LA, de Leeuw JR. Screen time associated with health behaviors and outcomes in adolescents. Am J Health Behav. 2013;37(6):819-30.

13. Nakamura PM, Teixeira IP, Papini CB, de Lemos N, Nazario MES, Kokubun E. Educação física escolar, atividade desportiva e atividade física total em adolescentes. Rev Bras Cineantropom Desempenho Hum. 2013;517-26.

14. Farias Júnior JC, Lopes AS, Motta J, Hallal PC. Prática de atividade física e fatores associados em adolescentes no Nordeste do Brasil. Rev Saúde Pública 2012;46(3):505-15.

15. Dias PJP, Domingos IP, Ferreira MG, Muraro AP, Sichieri R, Gonçalves-Silva RMV. Prevalência e fatores associados aos comportamentos sedentários em adolescentes. Rev Saúde Pública. 2014;48(2):266-74.

16. Santos A, Andaki ACR, Amorim PRDS, Mendes EL. Fatores associados ao comportamento sedentário em escolares de 9-12 anos de idade. Motriz Rev Educ Fís (Impr). 2013;19(3):25-34.

17. Rivera IR, Silva MA, Silva RD, Oliveira BA, Carvalho AC. Physical inactivity, TVwatching hours and body composition in children and adolescent. Arq Bras Cardiol. 2010;95(2):159-65.

18. Silva DAS, Tremblay MS, Gonçalves ECA, Silva RJS. Television Time among Brazilian Adolescents: Correlated Factors are Different between Boys and Girls. The Scientific World Journal. 2014;(2014):794539.

19. Tenório MCM, Barros MD, Tassitano RM, Bezerra J, Tenório JM, Hallal PC. Atividade física e comportamento sedentário em adolescentes estudantes do ensino médio. Rev Bras Epidemiol. 2010;13(1):105-17.

20. Associação Nacional de Empresas de Pesquisa. Critério de classificação econômica Brasil. São Paulo: Associação Nacional de Empresas de Pesquisa (dados com base no levantamento sócio econômico 2009); 2011.

21. Crocker PR, Bailey DA, Faulkner RA, Kowalski KC, Mcgrath R. Measuring general levels of physical activity: Preliminary evidence for the Physical Activity Questionnaire for Older Children. Med Sci Sports Exer. 1997;29(10):1344-9.

22. Kowalski K, Crocker P, Faulkner R. Convergent validity of the physical activity questionnaire for adolescents. Pediatr Exerc Sci. 1997;9(4):342-52.

23. Dumith S. Atividade física e sedentarismo: diferenciação e proposta de nomenclatura. Rev Bras Ativ Fís Saúde. 2010;15(4):253-4.

24. Gordia AP, Quadros TMBD, Campos WD, Petroski EL. Nível de atividade física em adolescentes e sua associação com variáveis sociodemográficas. Rev Port Ciên Desp. 2010;10(1):172-9.

25. Alves CFA, Silva RDCR, Assis AMO, de Oliveira Souza C, de Jesus Pinto E, Frainer DES. Fatores associados à inatividade física em adolescentes de 10-14 anos de idade, matriculados na rede pública de ensino do município de Salvador, BA. Rev Bras Epidemiol. 2012;15(4):858-70.

26. Hallal PC, Bertoldi AD, Gonçalves H, Victora CG. Prevalência de sedentarismo e fatores associados em adolescentes de 10-12 anos de idade. Cad Saúde Pública. 2006;22(6):1277-87.

27. Oliveira TCD, Silva AAMD, Santos CDJND, Silva JS, Conceição SIOD. Atividade física e sedentarismo em escolares da rede pública e privada de ensino em São Luís. Rev Saúde Pública. 2010;44(6):996-1004.

28. Dumith S, Marlos R Domingues MR, Gigante DP, Hallal PC, Menezes AMB, Kohl HW. Prevalence and correlates of physical activity among adolescents from Southern Brazil. Rev Saúde Pública 2010;44(3):457-67.

29. Ceschini FL, Andrade DR, Oliveira LC, Araújo Júnior JF, Matsudo VKR. Prevalence of physical inactivity and associated factors among high school students from state's public schools. J Pediatr. 2009;85(4):301-6.

30. Guedes DP, Guedes JE. Physical activity, cardiorespiratory fitness, dietary content, and risk factors that cause a predisposition towards cardiovascular disease. Arq Bras Cardiol 2001; 77(3): 243- 57.

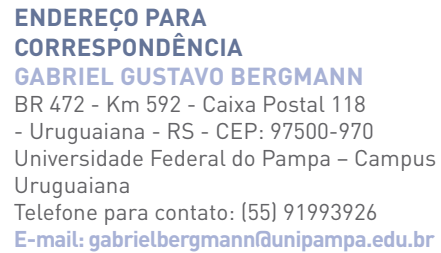

